\title{
Distribution of energy flow by dielectric waveguide with rhombic dielectric structure along a middle layer
}

\author{
Ryosuke Ozaki ${ }^{a)}$ and Tsuneki Yamasaki ${ }^{\text {b) }}$ \\ Department of Electrical Engineering, College of Science and Technology, Nihon \\ University, 1-8-14 Surugadai, Kanda, Chiyoda-ku, Tokyo 101-8308, Japan \\ a)ozaki@ele.cst.nihon-u.ac.jp \\ b)yamasaki@ele.cst.nihon-u.ac.jp
}

\begin{abstract}
In this paper, we analyzed the guiding problem for dielectric waveguide introduced as defect layers composed of dielectric circular cylinders with rhombic dielectric structure along a middle layer, and investigate the distribution of energy flow of dielectric waveguides for both $T M_{0}$ and $T E_{0}$ modes by using the propagation constants at the guided area. From numerical results, we can be obtained the confinement efficiency by loaded with rhombic dielectric structure compared with dielectric triangular cylinders for $T M_{0}$ mode. In the case of $T E_{0}$ mode, it is shown obviously that we can be obtained the confinement efficiency loaded with dielectric circular cylinders compared with rhombic dielectric structure.
\end{abstract}

Keywords: distribution of energy flow, dielectric waveguide, middle layer, rhombic dielectric structure

Classification: Electromagnetic theory

\section{References}

[1] J. D. Jannopoulos, R. D. Meade, and J. N. Winn, Photonic Crystals, Princeton University Press, Princeton, 1995.

[2] K. Sakoda, Optical Properties of Photonic Crystals, 2nd edition, SpringerVerlag, 2005.

[3] K. Yasumoto ed., Electromagnetic Theory and Applications for Photonic Crystals, CRC Press, 2005.

[4] Y. Nakatake and K. Watanabe, "Numerical analysis of two-dimensional photonic crystal waveguides formed by circular cylinders using periodic boundary conditions," IEICE Trans. Electron., vol. E94-C, no. 1, pp. 3238, Jan. 2011.

[5] A. Adibi, Y. Xu, R. K. Lee, A. Yariv, and A. Scherer, "Properties of the slab modes in photonic crystal optical waveguides," J. Light. Tech., vol. 18, no. 11, pp. 1554-1564, Nov. 2000.

[6] R. Ozaki and T. Yamasaki, "Propagation characteristics of dielectric waveguides with arbitrary inhomogeneous media along the middle layer," IEICE Trans. Electron., vol. E95-C, no. 1, pp. 53-62, Jan. 2012.

[7] R. Ozaki and T. Yamasaki, "Dielectric waveguide with arbitrary inhomogeneous media in the middle layer-propagation characteristics and 
distribution of energy flow-," The Papers of Technical Meeting on Electromagnetic Theory, IEE Japan, EMT-11-116, pp. 61-66, Nov. 2011 (in Japanese).

[8] R. Ozaki and T. Yamasaki, "Propagation characteristics and distribution of energy flow of dielectric waveguide with deformed rhombic dielectric structure in the middle layer," The Papers of Technical Meeting on Electromagnetic Theory, IEE Japan, EMT-12-15, pp. 57-62, Jan. 2012 (in Japanese).

\section{Introduction}

Light propagation in periodic structure waveguide such as photonic crystal waveguides is both theoretical and practical interest in many areas of physics and engineering. Applications include integrated optical circuit, optical resonator, and other optical devices $[1,2]$. Consequently, in the design of photonic crystal structures with periodic constants identical to the optical wavelength, it is important to investigate the stop band region or photonic band-gaps. However, though it is not analyzed the propagation constants in detailed in Bragg region, many numerical results are shown only the distribution of the electromagnetic fields by using the FDTD method or another numerical techniques $[3,4,5]$.

In previous paper [6], we analyzed the propagation characteristics of dielectric waveguide composed of dielectric circular cylinders array loaded with dielectric circular cylinders or dielectric triangular cylinders along a middle layer. In addition, we also investigated the distribution of energy flow at the guided area for the case of dielectric circular cylinders and dielectric triangular cylinders along a middle layer for both $\mathrm{TE}_{0}$ and $\mathrm{TM}_{0}$ modes. As these results, we denoted that it can be concentrated the energy by loaded with dielectric circular cylinders along a middle layer for $\mathrm{TE}_{0}$ mode, and loaded with dielectric triangular cylinders along a middle layer for $\mathrm{TM}_{0}$ mode.

However, the distribution of energy flow in the case of asymmetric structure such as triangular cylinders cannot be obtained sufficient results rather than that of symmetric structure with dielectric circular cylinders for $T M_{0}$ mode. On the other hand, the confinement effects in the defect area cannot be obtained compared with dielectric circular cylinders for $T E_{0}$ mode.

Consequently, we considered that the energy is carried outside by the influence of asymmetric structure [6].

In this paper, we have analyzed the distribution of energy flow for dielectric waveguide introduced as defect layers composed of dielectric circular cylinders loaded with symmetric structure such as rhombic dielectric structure along a middle layer for both $T M_{0}$ and $T E_{0}$ modes by using the propagation constants of the guided area, and investigated the effect of rhombic dielectric structure compared with dielectric circular cylinders for $T E_{0}$ mode and dielectric triangular cylinders for $T M_{0}$ mode $[7,8]$, respectively. As numerical results, it is shown that we can be obtained the effectiveness of 
proposed analytical model for $T M_{0}$ mode.

\section{Method of analysis}

We consider the dielectric waveguide with rhombic dielectric structure along a middle layer as shown in Fig. 1 (a). The structure $(D=L d)$ shown in the figure is periodic with a period $p$ along the $z$ direction and uniform in the $y$-direction. The upper region $S_{1}$ and lower region $S_{3}$ are defined by the dielectric constants $\varepsilon_{0}$. The configuration shown $(L=5)$ has dielectric circular cylinders with radius $a$ and $d_{1} / 2$ in the $x$ - and $z$-directions, respectively. The thickness of each layer is defined by $d$. The circular cylinder array in the periodic length is assumed to be dielectric constants $\varepsilon_{a}, \varepsilon_{b}$, and $\varepsilon_{3}$. The middle layer region has only rhombic dielectric structure with parameters $b$ and $c$ in the $x$ - and $z$-directions and dielectric constants $\varepsilon_{3}^{(m)}$. The permeability is assumed to be $\mu_{0}$ in all regions. The time factor $\exp (-i \omega t)$ in the field expression will be omitted.

In the formulation, the TM mode (the magnetic field has only the $y$ component) is discussed, and TE mode (the electric field has only the $y$ -

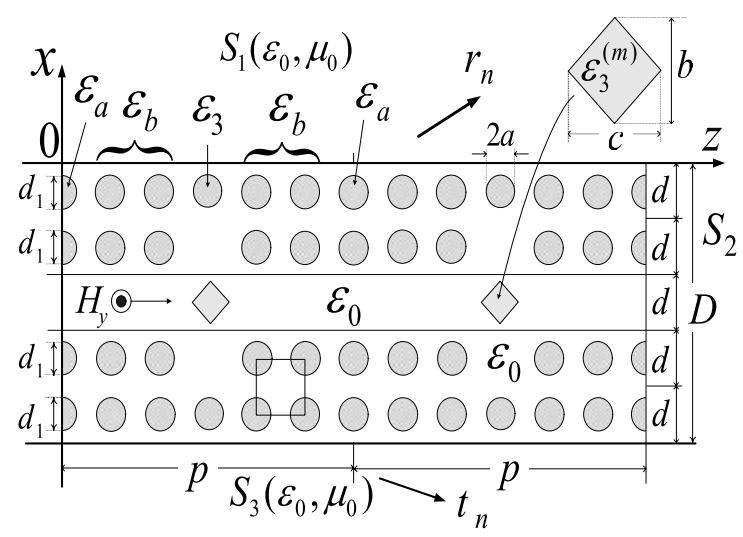

(a) Coordinate system

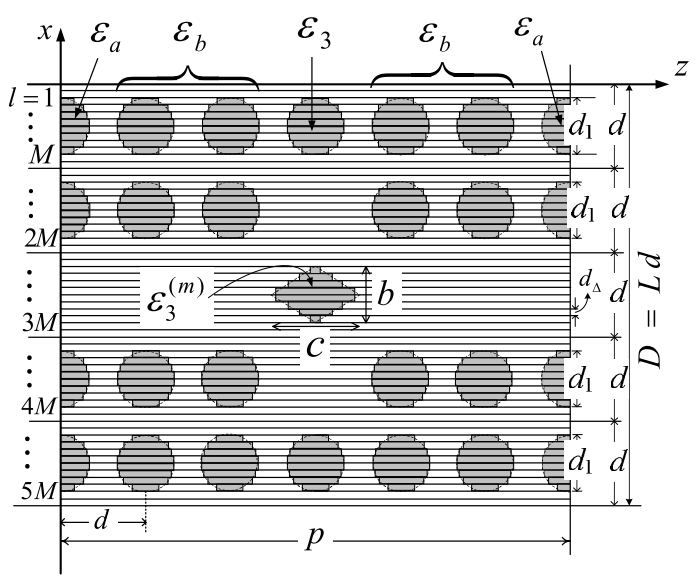

(b)Approximated multilayer region

Fig. 1. Structure of dielectric waveguide with rhombic dielectric structure along a middle layer 
component) only numerical results are presented. The magnetic fields in the regions $S_{1}(x \geq 0)$ and $S_{3}(x \leq-D)$ are expressed as

$$
\begin{aligned}
& H_{y}^{(1)}=e^{i \gamma z} \sum_{n=-N}^{N} r_{n} \exp \left(i k^{(n)} x+i 2 n \pi z / p\right), \\
& H_{y}^{(3)}=e^{i \gamma z} \sum_{n=-N}^{N} t_{n} \exp \left\{-i k^{(n)}(x+D)+i 2 n \pi z / p\right\}, \\
& k^{(n)} \triangleq \sqrt{k_{0}^{2}-(\gamma+2 n \pi / p)^{2}}, \quad k_{0} \triangleq 2 \pi / \lambda,
\end{aligned}
$$

where $\gamma(\triangleq \beta+i \alpha)$ and $k^{(n)}$ are the propagation constants in the $z$-direction and $x$-direction, respectively. The attenuation constants $\alpha$ is positive, $\lambda$ is the wavelength in free space, and $k_{0}$ is the wave number. $r_{n}$ and $t_{n}$ are unknown coefficients to be determined from boundary conditions. The sign of $k^{(n)}$ are given by the radiation condition in guiding problem [6]. In the region $S_{2}(-D<x<0)$, the first layer $(-d<x<0)$ is divided into $M$ thin layers and the profile in each layer $\left(d_{\Delta} \triangleq d / M\right)$ is approximated by step index profile $\varepsilon(z)$ as shown in Fig. 1(b). By using the eigenvalue $h_{\nu}^{(l)}$ and eigenvector $u_{\nu, n}^{(l)}$ founded from eigenvalue equation [7, 8], the electromagnetic fields can be expanded as finite Fourier series.

$$
\begin{aligned}
& H_{y}^{(2, l)}=\sum_{\nu=1}^{2 N+1}\left[A_{\nu}^{(l)} e^{-i h_{\nu}^{(l)}\left\{x+(l-1) d_{\Delta}\right\}}+B_{\nu}^{(l)} e^{i h_{\nu}^{(l)}\left(x+l d_{\Delta}\right)}\right] f_{\nu}^{(l)}(z), \\
& f_{\nu}^{(l)}(z) \triangleq e^{i \gamma z} \sum_{n=-N}^{N} u_{\nu, n}^{(l)} e^{i \frac{2 n \pi z}{p}}, \quad 1 \leq l \leq M \\
& E_{x}^{(2, l)} \triangleq \frac{1}{i \omega \varepsilon(z)} \frac{\partial H_{y}^{(2, l)}}{\partial z}, \quad E_{z}^{(2, l)} \triangleq \frac{-1}{i \omega \varepsilon(z)} \frac{\partial H_{y}^{(2, l)}}{\partial x}
\end{aligned}
$$

where $A_{\nu}^{(l)}, B_{\nu}^{(l)}$ are unknown coefficients to be determined from boundary conditions. Using the boundary conditions at $x=-l d_{\Delta}(l=1 \sim M-1)$, we can be obtained the matrix relation between $\mathbf{A}^{(1)}, \mathbf{B}^{(1)}$ and $\mathbf{A}^{(M)}, \mathbf{B}^{(M)}[6]$.

$$
\begin{aligned}
\left(\begin{array}{l}
\mathbf{A}^{(1)} \\
\mathbf{B}^{(1)}
\end{array}\right) & =\left(\begin{array}{ll}
\mathbf{S}_{1}^{(1)} & \mathbf{S}_{2}^{(1)} \\
\mathbf{S}_{3}^{(1)} & \mathbf{S}_{4}^{(1)}
\end{array}\right)\left(\begin{array}{ll}
\mathbf{S}_{1}^{(2)} & \mathbf{S}_{2}^{(2)} \\
\mathbf{S}_{3}^{(2)} & \mathbf{S}_{4}^{(2)}
\end{array}\right) \cdots\left(\begin{array}{ll}
\mathbf{S}_{1}^{(M)} & \mathbf{S}_{2}^{(M)} \\
\mathbf{S}_{3}^{(M)} & \mathbf{S}_{4}^{(M)}
\end{array}\right)\left(\begin{array}{l}
\mathbf{A}^{(M)} \\
\mathbf{B}^{(M)}
\end{array}\right), \\
& =\left(\begin{array}{ll}
\mathbf{S}_{1} & \mathbf{S}_{2} \\
\mathbf{S}_{3} & \mathbf{S}_{4}
\end{array}\right)\left(\begin{array}{l}
\mathbf{A}^{(M)} \\
\mathbf{B}^{(M)}
\end{array}\right)
\end{aligned}
$$

where matrix elements $\mathbf{S}_{1} \sim \mathbf{S}_{4}$ are given by following elements [6]:

$$
\mathbf{S}_{k}^{(l)} \triangleq\left[{ }^{(l)} s_{\nu, n}^{(k)}\right] ; \quad k=1 \sim 4, \quad 1 \leq l \leq M
$$

For the first layers to the middle layer, we can get the matrix relation between $\mathbf{A}^{(1)}, \mathbf{B}^{(1)}$ and $\mathbf{A}^{(3 M)}, \mathbf{B}^{(3 M)}$ as following equation [6]:

$$
\left(\begin{array}{l}
\mathbf{A}^{(1)} \\
\mathbf{B}^{(1)}
\end{array}\right)=\left(\begin{array}{ll}
\mathbf{S}_{1}^{(U)} & \mathbf{S}_{2}^{(U)} \\
\mathbf{S}_{3}^{(U)} & \mathbf{S}_{4}^{(U)}
\end{array}\right)\left(\begin{array}{l}
\mathbf{A}^{(3 M)} \\
\mathbf{B}^{(3 M)}
\end{array}\right) .
$$


Similarly, in the lower two layers $(-D<x<-3 d)$, we can be obtained the matrix relation as following equation [6]:

$$
\left(\begin{array}{l}
\mathbf{A}^{(5 M)} \\
\mathbf{B}^{(5 M)}
\end{array}\right)=\left(\begin{array}{ll}
\mathbf{S}_{1}^{(L)} & \mathbf{S}_{2}^{(L)} \\
\mathbf{S}_{3}^{(L)} & \mathbf{S}_{4}^{(L)}
\end{array}\right)\left(\begin{array}{l}
\mathbf{A}^{(3 M)} \\
\mathbf{B}^{(3 M)}
\end{array}\right) .
$$

Rearranging the unknown coefficients with respect to $\mathbf{A}^{(3 M)}$ in the middle layer, substituting Eqs. (8) and (9) into the boundary conditions at $x=0$ and $x=-D$, we can be obtained the following equation [6]:

$$
\mathbf{W} \cdot \mathbf{A}^{(3 M)}=\mathbf{0}
$$

where matrix element $\mathbf{W}$ is coefficients matrix [6].

For a nontrivial solution to exist in Eq. (10), we have the following characteristics equation [6]:

$$
\operatorname{det}(\mathbf{W})=0
$$

The propagation constants $\gamma$ can be found by utilizing the Muller's method [6] to calculate in Eq. (11).

To analyze the distribution of energy flow by using the propagation constant $\gamma$ founded from in Eq. (11), the Poynting vector is defined by following equation:

$$
\mathbf{S} \triangleq \mathbf{a}_{x} S_{x}+\mathbf{a}_{z} S_{z}
$$

where $\mathbf{a}_{x}$ and $\mathbf{a}_{z}$ are the unit vector in the $x$ and $z$-direction.

In the electromagnetic fields of the middle layer, the unknown coefficients are given by the ratio of $A_{\nu \neq 1}^{(l)} / A_{\nu=1}^{(l)}$ from Eq. (10). Therefore, we normalized coefficients $A_{\nu=1}^{(l=(M+1) / 2)}=1$ as following equation:

$$
\begin{aligned}
& \left\{\begin{array}{l}
H_{y}^{(2, l)} \\
E_{y}^{(2, l)}
\end{array}\right\}=\sum_{\nu=1}^{2 N+1}\left[A_{\nu}^{(l)} e^{-i h_{\nu}^{(l)}\left\{x+(l-1) d_{\Delta}\right\}}+B_{\nu}^{(l)} e^{i h_{\nu}^{(l)}\left(x+l d_{\Delta}\right)}\right] f_{\nu}^{(l)}(z) ; \\
& l=\frac{M+1}{2} .
\end{aligned}
$$

Then, $A_{\nu \neq 1}^{(l \neq(M+1) / 2)}$ and $B_{\nu}^{(l \neq(M+1) / 2)}$ of the unknown coefficient for the electromagnetic fields can be founded by solving the simultaneous equation at the center layer of the middle layer. In the case of another layer for the middle layer, unknown coefficients of the electromagnetic fields can be obtained by using the boundary conditions. Similarly, in the case of upper region $(-2 d<x<0)$, it is obtained from Eqs. (7) and (8). In the case of lower region $(-D<x<-3 d)$, electromagnetic fields can be used to those of the upper region from the symmetric structure. To obtain the distribution of energy flow, the electric fields founded from Eq. (6) are given by

$$
\begin{aligned}
E_{x}^{(2, l)}= & \frac{e^{i \gamma z}}{\omega \varepsilon(z)} \sum_{\nu=1}^{2 N+1}\left[A_{\nu}^{(l)} e^{-i h_{\nu}^{(l)}\left\{x+(l-1) d_{\Delta}\right\}}\right. \\
& \left.+B_{\nu}^{(l)} e^{i h_{\nu}^{(l)}\left\{x+l d_{\Delta}\right\}}\right] \sum_{n=-N}^{N} u_{\nu, n}^{(l)}(\gamma+2 n \pi / p) e^{i \frac{2 n \pi z}{p}}
\end{aligned}
$$




$$
\begin{aligned}
E_{z}^{(2, l)}= & \frac{e^{i \gamma z}}{\omega \varepsilon(z)} \sum_{\nu=1}^{2 N+1} h_{\nu}^{(l)}\left[A_{\nu}^{(l)} e^{-i h_{\nu}^{(l)}\left\{x+(l-1) d_{\Delta}\right\}}\right. \\
& \left.-B_{\nu}^{(l)} e^{i h_{\nu}^{(l)}\left\{x+l d_{\Delta}\right\}}\right] \sum_{n=-N}^{N} u_{\nu, n}^{(l)} e^{i \frac{2 n \pi z}{p}}
\end{aligned}
$$

Therefore, using the Eqs. (14) and (15), $x$-component and z-component of Poynting vector are given by following equations for both $T M$ and TE modes:

$$
\begin{aligned}
& S_{x}^{(T M)} \triangleq \operatorname{Re}\left[E_{z}^{(2, l)} \times\left(H_{y}^{(2, l)}\right)^{*}\right] / 2, \quad S_{z}^{(T M)} \triangleq \operatorname{Re}\left[E_{x}^{(2, l)} \times\left(H_{y}^{(2, l)}\right)^{*}\right] / 2, \\
& S_{x}^{(T E)} \triangleq \operatorname{Re}\left[E_{y}^{(2, l)} \times\left(H_{z}^{(2, l)}\right)^{*}\right] / 2, \quad S_{z}^{(T E)} \triangleq \operatorname{Re}\left[E_{y}^{(2, l)} \times\left(H_{x}^{(2, l)}\right)^{*}\right] / 2 .
\end{aligned}
$$

Here, the superscripts (TM) and (TE) indicate the TM and TE modes cases, respectively. The distribution of energy flow in the numerical analysis are given by

$$
P^{(T M, T E)} \triangleq \sqrt{\left\{S_{x}^{(T M, T E)}\right\}^{2}+\left\{S_{z}^{(T M, T E)}\right\}^{2}}
$$

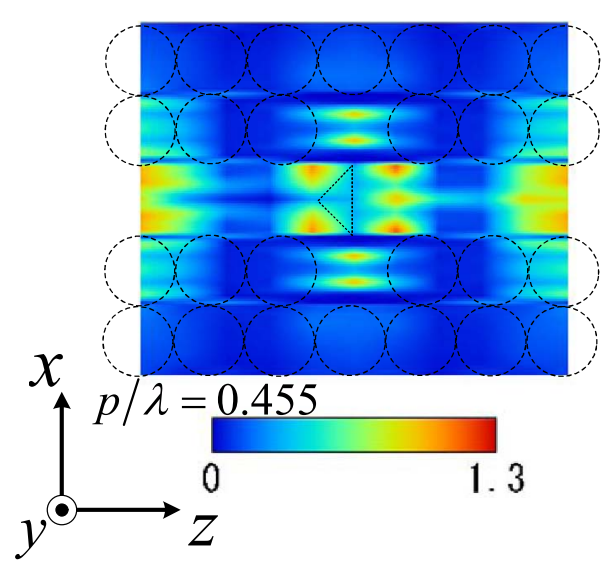

(a) dielectric triangular cylinder

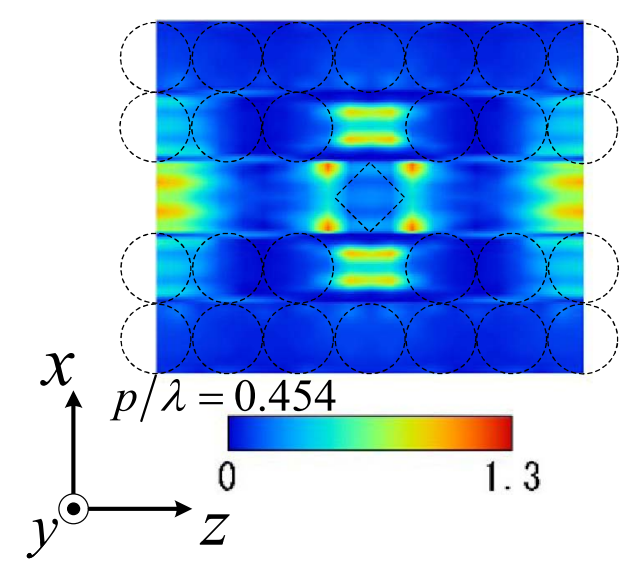

(b) rhombic dielectric structure

Fig. 2. Distribution of energy flow $P^{(T M)}$ at the guided area for $T M_{0}$ mode

\section{Numerical results}

We consider the lowest guided $T M_{0}$ and $T E_{0}$ modes $(0<p / \lambda<0.5)$, and the structure based on the circular cylinders $(c / p=b / p=1 / 6)$ as rhombic dielectric structure in the middle layer. The values of parameters chosen are $\varepsilon_{a} / \varepsilon_{0}=3, \varepsilon_{b} / \varepsilon_{0}=3, \varepsilon_{3} / \varepsilon_{0}=3, d_{1} / d=1, D / p=5 / 6,2 a / d_{1}=1, c / p=1 / 6$, $b / p=1 / 6$. The numerical computation in this paper is performed using the parameter $N=10$ or $N=9$ for $T E$ and $T M$ modes and $M=40$ which make the relative error to the extrapolated true values less than about $1 \%$ [6]. We use the excited normalized frequency $p / \lambda$ at the guided area of $\varepsilon_{3}^{(m)} / \varepsilon_{0}=3$ with the stop band region of $\varepsilon_{3}^{(m)} / \varepsilon_{0}=1[6]$.

Figures $2(\mathrm{a})$ and $2(\mathrm{~b})$ show the distribution of the energy flow $P^{(T M)}$ at the guided area for the case of loaded with dielectric triangular cylinder as 
excited normalized frequency $p / \lambda=0.455$ and rhombic dielectric structure as excited normalized frequency $p / \lambda=0.454$ in the middle layer as a condition of $\varepsilon_{3}^{(m)} / \varepsilon_{0}=3$ for $T M_{0}$ mode, respectively.

From Figs. 2 (a) and 2 (b), we can see the following features:

(1) The energy of defect area for rhombic dielectric structure is stronger than that of dielectric triangular cylinder.

(2) The middle layer region loaded with the rhombic dielectric structure instead of dielectric triangular cylinder can be obtained distribution of symmetry.

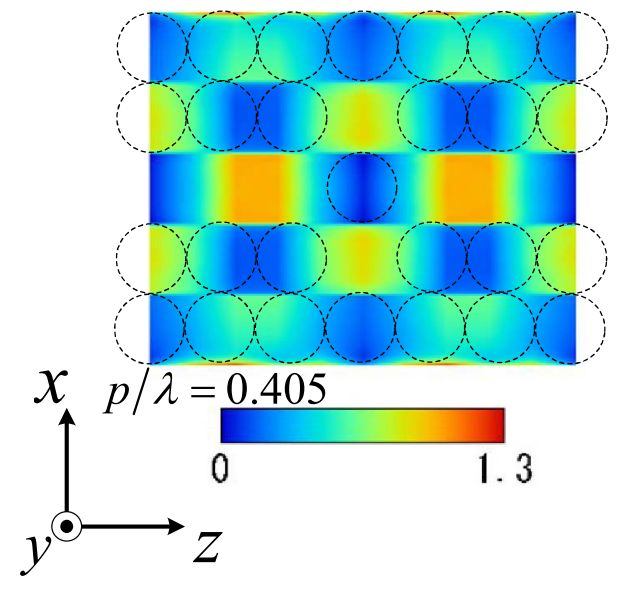

(a) dielectric circular cylinder

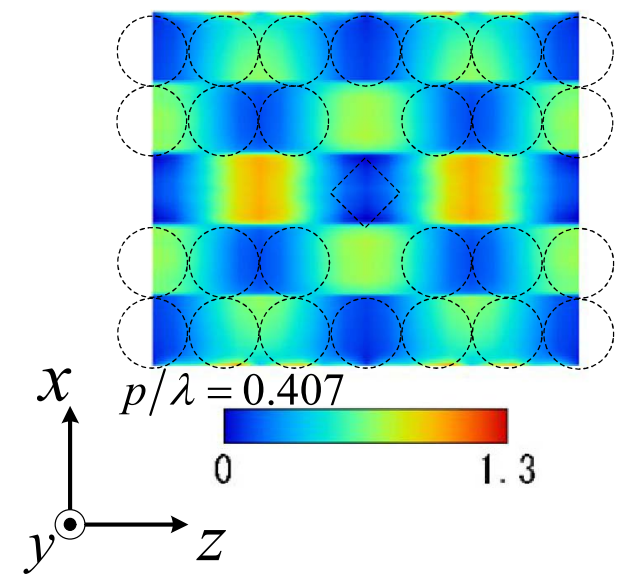

(b) rhombic dielectric structure

Fig. 3. Distribution of energy flow $P^{(T E)}$ at the guided area for $T E_{0}$ mode

Figures $3(\mathrm{a})$ and $3(\mathrm{~b})$ show the distribution of energy flow $P^{(T E)}$ at the guided area for the case of loaded with dielectric circular cylinders as excited normalized frequency $p / \lambda=0.405[6]$ and rhombic dielectric structure as $p / \lambda=0.407$ in the middle layer as same condition of Fig. 2 for $T E_{0}$ mode, respectively. From Figs. 3 (a) and 3 (b), we can see the following features:

(1) The energy of defect area for dielectric circular cylinder is strong compared with rhombic dielectric structure.

(2) In the outside region of the defect area, the distribution of energy flow is similar to the case of dielectric circular cylinder.

\section{Conclusions}

In this paper, we analyzed the guiding problem for dielectric waveguide introduced as defect layers composed of dielectric circular cylinders array with symmetric structure such as rhombic dielectric structure along a middle layer, and investigated the distribution of energy flow of dielectric waveguides for both $T M_{0}$ and $T E_{0}$ modes by using the propagation constants at the guided area.

Numerical results are given for the influence of the middle layer loaded with rhombic dielectric structure in terms of the distribution of energy flow. 
As numerical results, we can be obtained the confinement efficiency loaded with rhombic dielectric structure compared with dielectric triangular cylinder for $T M_{0}$ mode. In the case of $T E_{0}$ mode, it is shown obviously that we can be obtained the confinement efficiency loaded with dielectric circular cylinder compared with rhombic dielectric structure.

In the future, we will be investigated the influence of convergence for large periodic length and optimum shape in the middle layer. 